\title{
Social determinants of tobacco use: towards an equity lens approach
}

\author{
Alexios-Fotios A. Mentis ${ }^{I}$
}

\begin{abstract}
Tobacco is the only commercial product that eventually kills nearly half of all long-term users. The prevalence of tobacco use is disproportionately high in lower socioeconomic strata and vulnerable groups (such as adolescents) within and across countries. Given its highly addictive nature, tobacco use perpetuates poverty and loss of opportunities, thus undermining the UN Sustainable Development Goals. Moreover, by shaping the national and international context, globalization and governance impact on the tobacco epidemic and underlying disparities. Therefore, socio-economic gradients, which influence predisposition to tobacco uptake and cessation, must be confronted. Here I argue that tobacco prevention and control must be addressed through a lifelong, equity lens approach. This approach describes the essential need for every individual to have equal access to informative prevention and cessation services independent of income, occupational status, social stratum, or residence. I also contend that rather than being occupied with "research on research", the focus should shift to how to practically implement the existing accumulated, cogent body of scientific evidence in a societally equitable manner. Finally, in line with the core dilemma of "who really governs the policies that shape our health?" raised by the WHO's Director General, it is time for civil society either on its own or in partnership with local authorities to formulate policies that implement the "health for all" imperative rather than the currently dominant "wealth for some".
\end{abstract}

\author{
AFFILIATION \\ 1 The Johns Hopkins \\ University Advanced Academic \\ Programs (AAP), Baltimore, \\ USA \\ KEYWORDS \\ Tobacco; Social Determinants \\ of Health; Health Equity; Public \\ Health; Development Agenda; \\ Globalization; Governance. \\ CORRESPONDING AUTHOR \\ Alexios-Fotios A. Mentis, \\ The Johns Hopkins University, \\ AAP \\ 3400 North Charles Street - \\ Wyman Park Building S - 612 \\ Baltimore, MD 21218 \\ United States of America \\ E-mail: amentis1@jhu.edu
}

\section{INTRODUCTION}

Tobacco use fulfils all the criteria to be addressed as a World Health Organization (WHO) Priority Public Health Condition $^{1}$ : (i) it is an epidemic that significantly contributes to the aggregate burden of non-communicable diseases (NCDs); (ii) its incidence and severity have dramatically shifted from developed to resource-poor countries; and (ii) there are predominant inter- and intra-national inequities. With the exception of Africa, where infectious diseases still predominate, all other regions are burdened with chronic non-communicable diseases (NCDs) that are leading causes of death ${ }^{2}$. Of the four top health risks (tobacco use, harmful alcohol consumption, physical inactivity, and obesity), tobacco use is consistently ranked first with respect to its contribution to the aggregate burden of $\mathrm{NCDs}^{3}$. Smokers prematurely lose almost two decades of life ${ }^{3}$, and the projected global prevalence of tobacco-related causes of death is estimated to be around $45 \%$ by 2030 .

Inter-regional disparities are most discernible in low- and middle-income countries (LMICs), notably of Central and Eastern Europe and South-East Asia ${ }^{4,5}$. Male children and young people are most at risk for initiating and continuing tobacco use $\mathrm{e}^{6-8}$. When compounded by other determinants such as income and social disadvantage, disparities in initiation and continuation become even starker.

Therefore, since: (i) tobacco is so strongly connected to health, and (ii) "health is a state of wellbeing emergent from conducive interactions between individuals' potentials, life's demands, and social and environmental determinants" as per the Meikirch model of health, here I focus on how 
social determinants of tobacco use affect the health of the most vulnerable in society. More precisely, I discuss how inequity related to social, economic, and political determinants (including gender, age, income, governance, and globalization), which collectively form the basis of the health impact pyramid ${ }^{10}$, leads to discrepancies in tobacco use and impedes its cessation and control. In doing so, I provide further insight on how "an individual's smoking trajectory is the accumulation of social disadvantage over the entire life-course"11. Each determinant is critically summarised, and, although they are ultimately interconnected, describe unique characteristics that contribute to a more unified approach. Only by addressing such underlying gradients of the tobacco epidemic will health systems be able to meet the particular needs of different groups, not least the most disadvantaged.

\section{Socio-economic determinants and tobacco use: income, occupation and social class}

Individuals in lower income quintiles display higher smoking rates compared to those in upper income quintiles. These disparities are even starker and will be exacerbated in the future in LMICs, which are home to around $80 \%$ of the more than one billion smokers ${ }^{6}$. Poor households are estimated to spend around $10 \%$ of disposable income on tobacco if there is one smoker in the family ${ }^{12}$. Tobacco use is common in low income populations for two main reasons: (i) poverty negatively correlates with power and influence on policy formulation, thus leaving people practically voiceless, and (ii) unplanned, poverty-driven urbanization has resulted in the proliferation of slums and other informal settlements, where tobacco use is the norm ${ }^{14}$.

With respect to occupational status, itself a social determinant of health, the polarization of employed vs. unemployed, formal vs. informal sector, "blue-collar" workers vs. "white-collar" workers, and in-work poverty vs. highly remunerated jobs have a crucial impact on tobacco susceptibility, exposure, and use. Beyond its tight connection with income levels, occupational status is closely related to job security and insurance, i.e., access to health services. Work environments shape their own "pro-smoking" or "co-smoking" culture, defining availability and acceptability ${ }^{15,16}$. For example, "blue-collar" employees often work in outdoor spaces and have lower health literacy. Also, anecdotal evidence suggests that smoking is symbolic to them, e.g., sharing cigarettes during a break may represent a culture of friendly collegiality.

Different social classes tend to have different social networks that generate political empowerment and inclusion if upstream and social exclusion and isolation if downstream. With respect to health effects, it has consistently been shown that stronger social networks (either offline or online) are related to increased longevity ${ }^{17}$. As a biological correlate, social isolation (e.g., long-term unemployment, mental illness, homelessness, and displacement) are associated with higher fibrinogen levels, a marker for cardiovascular events ${ }^{18}$. Thus, it would be interesting to study the combined effects of social isolation and smoking on health status.

\section{Gender-based differentiation in tobacco use}

Gender-differentiated societal values and norms exercise an impact on tobacco prevalence ${ }^{19}$ and gender is intrinsic to sociological and epidemiological studies. Exposure to risk and vulnerability are common to both sexes, but the wider gender divide in prevalence calls for more research to establish the influencing variables such as childhood background, educational status, low social status, normative and descriptive influences, and ethnicity ${ }^{20}$. Global trends indicate that smoking is much more common in males than females in those older than 15 years $^{7}$, while gender differences are less striking in adolescents $^{21}$.

For male adults, the main gradients are a vicious cycle of low education and reduced income and the individual and transgenerational social psychology that imposes smoking as a model of masculinity ${ }^{4}$. Taken further, the Marlboro cowboy did a lot to promote sales but his premature death from lung cancer did little to positively influence the smoking habits of next generations ${ }^{22}$. Psychologists may yet shed light on how the feeling of having no control over life, which is rooted in aggregate social disadvantage, may predispose men to tobacco initiation and continuation, with smoking perceived as a means to cope with daily hardships and related distress of a person who lacks self-confidence ${ }^{16}$. This mind-set, tied with a lack of structural and social support networks, hinders most male smokers from abstaining from tobacco. For female smokers, apart from factors shared with male smokers such as low income and educational level, women often have to deal with early motherhood and anxiety to earn money to protect their children, which may negatively impact on their psychological health and make them more vulnerable to tobacco use $\mathrm{e}^{23}$.

With respect to LMICs, gender discrimination remains an influential gradient that can explain disparities in tobacco prevalence between different regions separately from other cultural, religious or ethnical norms ${ }^{24}$. For instance, both smoking and smokeless tobacco consumption is more common in men than in women in South Asia. By exploiting existing divergences, the tobacco industry seems to have aggressively and unashamedly targeted poor, uneducated women in LMICs 
through deceptive marketing campaigns ${ }^{25}$. For example, they have started to use lipstick or perfume bottle-like packaging and promote "slim" cigarettes in line with the "Be Slim" identity ${ }^{26}$. Future trends in female smoking are likely to be influenced by Tobacco Transnationals, who have "exploited gendered imagery and issues across cultures for decades" ${ }^{\prime 27}$.

\section{Adolescence: the "ethmoid sinus" of external influences}

As a vulnerable subpopulation undergoing rapid biological and psychosocial changes, adolescents are at significant risk of starting smoking. The reward-seeking regions of the brain develop earlier than those responsible for planning and emotional control, which leads to less critical thinking and more emotional impulsion ${ }^{28}$. Concurrently, the brains of adolescents have a remarkable capacity for adaptation to change; thus, tobacco use during this life period is falsely perceived as normative when, in fact, it is pathological. In contrast, continuation during young adulthood might be better attributed to nicotine addiction and barriers to access to cessation help, which prevail in most countries ${ }^{28}$.

Three micro-environments "co-shape" adolescent vulnerability or resistance to tobacco initiation: family, school, and choice of friends $\mathrm{s}^{29}$, upon which macro-environments, such as advertising and public policies, may exert exacerbating or mitigating roles. First, health attitudes and behaviours such as healthy diet, physical exercise, and learning to abstain from tobacco and harmful drinking are assimilated and shaped within the family environment during the early years of life. These results have been corroborated by Health Behaviour of School-aged Children (HBSC) surveys ${ }^{30}$. Notwithstanding the potential of this environment to act as an "incubator" for health promotion, the reality is much more discouraging. At least $40 \%$ of children grow up in families where one or even both parents use tobacco products, independent of parental educational level, child exploitation, and lack of parental support ${ }^{28}$. Hence, although parental control may wane over adolescence, parental role models continue to amplify any pre-existing adolescent predisposition to tobacco use. Although it is worth noting that that paternal smoking or that of other male family members exerts a greater influence than maternal smoking ${ }^{3}$, public health policies including anti-smoking social networks and campaigns still need to be addressed to all family members.

With respect to the school environment, the research focus has shifted from student performance toward school culture ${ }^{28}$ because it has the potential to formulate an ethos that can offset or enhance the influence of family and peers. Many students report having seen peers or school personnel smoking within the premises ${ }^{31}$. However, interactive health projects within schools can strengthen life skills and social competence, which build resistance to addictive behaviours while promoting positive health comportments. Moreover, they can provide support and guidance to parents or community leaders, so effectiveness may be widely spread ${ }^{32}$.

In parallel, the tobacco industry has been very creative in circumventing advertising bans aimed at adolescents by fully exploiting, if not manipulating, the film industry, especially those that are export-oriented (e.g., Bollywood) or where popular actors and actresses appear smoking ${ }^{33}$. The tobacco industry has been equally imaginative in circumventing other regulatory restrictions (e.g., sales on the spot by promoting on-line cigarette purchase). To this end, international agencies, national governments, and civil society must engage in resourceful and innovative de-normalisation and countermarketing tobacco conglomerates.

\section{Tobacco-related negative social gradients: a threat to the UN sustainable development agenda}

The described social determinants of tobacco initiation and use have "domino" effects. Even though most smokers recognise that "tobacco causes death", smokers tend to be oblivious to the fact that tobacco is the only commercial product that eventually prematurely kills nearly half of all long-term users. Tobacco-related premature death (the primary leading cause of premature deaths for men and the second, after blood pressure, for women) deprives households of their earners, increases healthcare costs for families, and causes further drainage to already weak health systems $\mathrm{s}^{34}$. In parallel, ill health and morbidity affect disposable income and fuel a downward poverty spiral, since poor households cannot afford to pay for medical treatment. It is now appreciated that many households have slipped under the poverty line due to tobacco-related diseases, especially in LMICs. $80 \%$ of smokers live in LMICs, where households and governments are disproportionately affected by these adverse consequences of tobacco use and cannot afford to meet increased incurred expenditure. Together, these factors constitute a threat to the UN sustainable development agenda ${ }^{35,36}$.

In addition, tobacco use imposes a transgenerational health, financial, and even environmental burden. For example, in some countries in which approximately half of children are stunted and around $40 \%$ are underweight, the prevalence of parental smoking is approximately $70 \%{ }^{37}$. Also, besides direct consequences on family income, there are other indirect impacts on a child's future prospects. Tobacco expenses deprive families of a source of income for education and health, two major "interrupters" of the vicious poverty cycle, 
hence amplifying the risk of trans-generational poverty ${ }^{38}$. Furthermore, environmental pollution from household tobacco use seriously jeopardizes children's health and that of female non-smokers, especially during pregnancy. Secondhand smoking is the "first major environmental risk factor of the unborn", and tobacco is detrimental to children's life-cycle health ${ }^{39}$. Fortunately, the WHO's work to protect and promote children's environmental health has been exemplary over the last few years ${ }^{40,41}$.

\section{Tobacco production: a violation to farmers' health and children's rights}

One way to tackle tobacco use and its society-wide effects is to confront tobacco production, which has its own social consequences. Its regulation in the broader context of framing trade liberalization is essential. Tobacco farming, mostly contracted and allegedly a lucrative income for poor farmers, undermines food security at the national level ${ }^{42}$, aggravates environmental degradation ${ }^{43}$, and severely damages family health and children's education. Indeed, children, adolescents, and women heavily engaged in the tobacco farming in LMICs as an unremunerated workforce are exposed to toxic pesticides and suffer from Green Tobacco Sickness ${ }^{35}$. Characteristic symptoms of this disease include vomiting, dizziness, and high blood pressure ${ }^{44}$, while the effects on maternal and foetal health remain less well investigated ${ }^{45}$. Nevertheless, reports from the Campaign for Tobacco-Free Kids have recorded unexplained miscarriages in female tobacco farmers and increased congenital abnormalities in their new-borns ${ }^{46}$. At the same time, exposure to large amounts of toxic agrochemicals, long since banned in developed countries, represents another example of vulnerability and accumulation of social disadvantage.

Moreover, children and adolescent girls engaged in farming or "bidi rolling" do not pursue education, thus losing the opportunity to offset the poverty trap. More often than not, the obligatory engagement of girls in bidi rolling intentionally deters them from schooling in several LMICs that display gender discrimination through social control ${ }^{19}$. For these reasons, trade agreements and capital investments ought to be deemed detrimental to global health and sustainable development, a statement indirectly corroborated by the World Bank $^{47}$. Countries should prioritise human health over trade liberalization by insisting that all involved parties take public responsibility ${ }^{48}$.

However, other socio-economic consequences have gone almost unnoticed and are beyond the reach of specialised international and non-governmental organizations agencies and politically engaged medical and public health scientists. Tobacco is the most widely grown non-food crop, with 80 LMICs producing over $80 \%$ of this global harvest. Dominant tobacco companies with subsidiaries in over sixty developing countries directly monopolize or control shares in tobacco monopolies ${ }^{49,50}$. In this manner, they exercise significant power on local political systems, influencing policymaking on tobacco control interventions or other regulatory schemes, which deeply impacts on child labour, hazardous occupational risks, and price setting for tobacco crops $^{51}$.

The policies of these companies allegedly comply with International Conventions such as the Worst Forms of Child Labour Convention in 1999 and the UN Covenant on Economic, Social, and Cultural Rights. However, this "compliance" is likely to be a euphemism, because large numbers of children work in farming or on-field processing in several countries, both LMICs and developed. In some countries, $60 \%$ of tobacco pickers are thought to be immigrant children, despite the tobacco companies' claims ${ }^{52}$. Surprisingly, children aged over 12 can work in tobacco fields for unlimited hours with permission from parents in some countries, while even 7-year-old children have been reported to be partially engaged with farming after school hours, with three-quarters among them reporting symptoms associated with acute nicotine poisoning ${ }^{53}$.In sub-Saharan Africa, the incidence of child labour in the informal sector, especially in tobacco and cotton farming, in some states represents $\sim 90 \%$ of children aged 5-14, at least until the end of the last decade ${ }^{54}$. These children are exposed to approximately $55 \mathrm{mg}$ of dissolved nicotine per day, which is equivalent to more than 30 cigarettes $^{44}$.

Although the evidence on the effect of such hazardous working conditions on physical and brain development has been presented to governments, representatives of the tobacco industry, and international agencies, child labour remains "the tobacco industry's smoking gun", and, undeniably, constitutes modern slavery. This slavery is reflected in "long hours, little pay, no school" and complete "rightlessness", as previously discussed for child migration ${ }^{36,56}$.

\section{Globalisation, trade liberalization, and health governance: effects on the tobacco arena}

Globalisation and health governance are critical determinants of tobacco use, given that they shape both macro- and microsocioeconomic contexts and thus have an effect on all clusters of social (dis-)advantage ${ }^{57}$. Globalisation can undeniably be beneficial to health, for instance by bridging gaps in access to pharmaceuticals or by increasing income through economic growth, in line with the necessary mechanisms for income 
redistribution. Nevertheless, the increasingly globalised production and uncontrolled marketing of harmful products such as tobacco undeniably pose threats to global health, an observation made two decades ag $0^{58}$.

In addition, globalisation generates broader policy concerns related to trade liberalisation, market de-regulation, and the withdrawal of the State from its regulatory role; all these can be translated to disparities across and within countries. The Secretary-General, addressing the UN Conference on Trade and Development in 2003, repeatedly stressed the need for market re-regulation, of States resuming their regulatory role, parity in shaping trade, and investment rules. LMICs attempted to regulate trade but political imbalances, as well as restricted resources and expertise, made the whole endeavour rather difficult ${ }^{59}$.

Having invaded many LMICs, the tobacco industry influences political elites and policy shaping through corporate social responsibility (CSR), whose prime target is to create corporate political activity within a country ${ }^{34,60,61}$. The latter has been camouflaged by CSR on health awareness projects for HIV, hepatitis, and unsafe water (among others) in LMICs to divert attention from the consequences of tobacco use. For example, the industry has aimed to project an environmentally friendly profile through CSR, according to which contracted farmers were required to plant Eucalyptus for their tobacco curing needs to avoid aggravating deforestation. Ironically, this tree is particularly water consuming, rapidly depleting water tables and scorching the soil ${ }^{51}$.

In addition, recent work by Ulucanlar et al. ${ }^{62}$ has very pointedly shed light on how "industry, working through different constituencies, constructs a metanarrative to argue that proposed policies will lead to a dysfunctional future of policy failure and widely dispersed adverse social and economic consequences. Simultaneously, it uses diverse, interlocking insider and outsider instrumental strategies to disseminate this narrative and enhance its persuasiveness in order to secure its preferred policy outcomes" ${ }^{\prime 2}$.

Governments of tobacco-producing countries have greater difficulty in confronting the industry since their foreign earnings are closely associated with it and many of their citizens are engaged in tobacco farming ${ }^{63}$. Global advocacy proponents and economists should assist these governments to become more cognisant of the net cost induced by the tobacco industry ${ }^{64}$ if all variables such as farmers' debt, impaired health, and environmental aggravation are (cumulatively) taken into consideration ${ }^{61}$. There is also a dire need for political impetus and support from international agencies for crop diversification, enforcement of regulatory mechanisms, and compliance with mandatory tobacco control and prevention measures.
Acting as an antidote to industry strategies are, fortunately, some LMICs, which - by embracing health as a productivity factor-have prioritised human health over trade profits. Policies towards these directions include (but are not limited to): (i) increased taxation to the highest level to the inconvenience of major tobacco industries, thereby curbing consumption but doubling tax revenues; (ii) official advice to contracted farmers to diversify into horticulture and other products to avoid impoverishment through debt and to protect their health; (iii) a government's "tough fight" against the tobacco industry's attempts to circumvent advertising bans by promoting its logo on coffee packs and franchising logoed coffee bistros (Chaloupka et al. 2012; Zhong 2007) ${ }^{59,65}$.

\section{From the necessity of legal frameworks towards achieving real-time implementation}

The WHO provides a comprehensive, legally binding platform for tobacco prevention and control. There are 180 signatories to the Framework Convention on Tobacco Control (FCTC), which is committed to reducing tobacco prevalence by $25 \%$ by 2025 . Following the FCTC, concrete measures have been put in place that will hopefully deliver the ultimate goal of mitigating tobacco-induced NCDs. Therefore, it provides a unique platform for resource-poor countries wishing to protect their populations' health over profits but lack the financial resources or legal expertise to act against tobacco conglomerates ${ }^{34}$.

The so-called "MPOWER" (i.e., Monitoring, Protect, Offer help, Warn about dangers, Enforce regulatory interventions, and Raise taxes) actions offer best-to-buy and effective mechanisms to reduce tobacco consumption ${ }^{66}$. According to the latest FCTC report, many countries have legislated for tobacco-free environments $(n=49)$, have imposed warning labels on packages $(n=42)$, have implemented mass-media campaigns against smoking $(n=39)$, have significantly raised taxes $(\mathrm{n}=33)$, have banned advertising $(\mathrm{n}=29)$, or have implemented cessation programmes $(n=24)^{67}$.

This progress should be commended but, given the tobacco epidemic, there is room for improvement. Deciphering the reasons why such instruments have not been adopted in other countries is pivotal. It seems that strained health systems, poor administrations incapable of enforcing restrictions, and the unwillingness of governments to diminish commercial interests could be underlying causes. To this end, global advocacy and increased development aid resources could partially offset obstacles. In addition, strengthening the implementation of specific FCTC articles, like the one on Intergovernmental Transparency (No. 5.3), Diversification 
(No. 17), and Occupational Health Hazards (No. 18) remains crucial.

\section{Suggestions for future action in societal settings against the tobacco epidemic}

Beyond the legal framework, there is a place for civil society to intervene as an outstanding player. Physician associations, NGOs, parents' associations, community opinion leaders, and other social networks can counteract corporate profits. In many countries, civil society collaborates with local, national or supranational entities to create effective social marketing against tobacco initiation and in favour of cessation. In 2005, the European Union launched an anti-smoking campaign called "For a life without tobacco" in its 28 member states ${ }^{34}$; its core message was that smoking impacts on the whole of society and not exclusively on smokers, calling the latter to change attitudes and behaviours on the basis of critical thinking. Many NGOs participated in the campaign, which proved successful in boosting smokers' intention to quit and reflect on the detrimental effects of their habit not only on themselves but wider society.

Efficient and cost-effective tools such as counter-marketing, de-normalisation, and social networks can also be utilized by civil society to support tobacco cessation. Social media can also be exploited to influence life skills and social competence to resist starting smoking. Interventions in school and community environments by paraprofessional educators have revealed truths, which have been systematically (and intentionally) obscured by the tobacco industry. Such tools seem to be very efficient with respect to out-reach and isolated communities, which have no other alternatives for information and education on preventative measures. The comparative advantages of such projects are multiple: primarily, they are interactive by nature and include parents, students, educators, and opinion leaders, who can generate chain reactions through such interactions. Additionally, they are cost-effective or even cost-free, needing neither infrastructure nor equipment.

At the national level, intervention projects in schools can be easily organised and implemented. In many European countries, such interactive projects that focus on building positive life skills are mandatory, following instructions issued by national authorities, and are delivered to children aged ten- to twelve- years old. Children living in out-reach areas in resource-poor countries are unlikely to have access to such preventative information. Hence, there is a dire need for civil society in this situation to undertake the role of the State with regard to protecting and warning against negative consequences, de-normalising to the greatest possible extent the "glamour" projected by tobacco industry through advertising or the film industry.

\section{CONCLUSIONS}

In conclusion, tobacco use and production have deep roots and ties with negative social determinants. The epidemic character of tobacco impedes both social and health equity and the UN Sustainable Development Goals ${ }^{15}$. Reversing such determinants, particularly in resource-poor settings, would first necessitate a radical societal transformation and political willingness that seems rather utopian given the complex global interdependencies described ${ }^{10}$. However, doing so would promote the "right to health" and the shared responsibility to respond both in the short- and long-term to the dilemma "health for all or wealth for a few?" and the imperative "health in all policies". Overall, "shared responsibilities contribute to shared benefits", and recognising this will help in the on-going war between those who want to protect people's health (including health organisations) and the tobacco conglomerates ${ }^{9}$.

\section{REFERENCES}

1. World Health Organization: Guidelines for implementation of Article 5.3 of the WHO Framework Convention on Tobacco Control on the protection of public health policies with respect to tobacco control from commercial and other vested interests of the tobacco industry. 2008a. Geneva: World Health Organization.

2. Lim SS, Vos T, Flaxman AD, Danaei G, Shibuya K, Adair-Rohani H, et al: A comparative risk assessment of burden of disease and injury attributable to 67 risk factors and risk factor clusters in 21 regions, 1990-2010: a systematic analysis for the Global Burden of Disease Study 2010. Lancet 2012, 380(9859):2224-60. doi: 10.1016/ S0140-6736(12)61766-8

3. World Health Organization: WHO report on the global tobacco epidemic, 2013a: enforcing bans on tobacco advertising, promotion and sponsorship. 2013a. Geneva: World Health Organization, Pages 38-41. 2013.

4. Jha P, Peto R, Zatonski W, Boreham J, Jarvis MJ, Lopez AD: Social inequalities in male mortality, and in male mortality from smoking: indirect estimation from national death rates in England and Wales, Poland, and North America. Lancet 2006, 368(9533): 367-70.

5. Mathers CD, Loncar D: Projections of global mortality and burden of disease from 2002 to 2030. PLoS Med 2006, 3(11): e442. doi: 10.1371/journal.pmed.0030442

6. Asma S, Mackay J, Song SY, Zhao L, Morton J, Palipudi KM, et al.: The GATS Atlas. 2015. CDC Foundation, Atlanta, GA.

7. King BA, Mirza SA, Babb SD: GATS Collaborating Group. A crosscountry comparison of secondhand smoke exposure among adults: findings from the Global Adult Tobacco Survey (GATS). Tob Control 2013, 22(4): e5. doi: 10.1136/tobaccocontrol-2012-050582

8. Warren CW, Jones NR, Peruga A, Chauvin J, Baptiste JP, Costa de Silva V, el Awa F, Tsouros A, Rahman K, Fishburn B, Bettcher DW, Asma S: Centers for Disease Control and Prevention (CDC). Global youth tobacco surveillance, 2000-2007. MMWR Surveill Summ 2008, 57(1): 1-28.

9. Bircher J, Kuruvilla S: Defining health by addressing individual, 
social, and environmental determinants: New opportunities for health care and public health. Journal of Public Health Policy. 2014, 35(3): 363-86. doi: 10.1057/jphp.2014.19

10. Garrett BE, Dube SR, Babb S, McAfee T: Addressing the social determinants of health to reduce tobacco-related disparities. Nicotine \& Tobacco Research 2015, 17(8):892-7. doi: 10.1093/ntr/ntu266

11. Kunst A, Giskes K, Mackenbach J: Socio-economic Inequalities in Smoking in the European Union: Applying an Equity Lens to Tobacco Control Policies: for the EU Network on Interventions to Reduce Socio-economic Inequalities in Health. 2004. Erasmus Medical Center Rotterdam.

12. World Health Organization: Systematic review of the link between tobacco and poverty. 2011. Geneva: WHO. Pages: 1-10, 21-34, 47-142. Geneva: World Health Organization.

13. Brian K: OECD Insights From Aid to Development: The Global Fight against Poverty: The Global Fight against Poverty. 2012. OECD Publishing.

14. Arora M, Tewari A, Tripathy V, Nazar GP, Juneja NS, Ramakrishnan L, Reddy KS: Community-based model for preventing tobacco use among disadvantaged adolescents in urban slums of India. Health Promotion International 2010, 25(2): 143-152. doi: 10.1093/ heapro/daq008

15. Marmot M, Friel S, Bell R, Houweling TA, Taylor S, \& Commission on Social Determinants of Health: Closing the gap in a generation: health equity through action on the social determinants of health. The Lancet 2008, 372(9650): 1661-1669.

16. Farrelly MC, Evans WN, Sfekas AE: The impact of workplace smoking bans: results from a national survey. Tobacco Control 1999, 8(3): 272-277.

17. Hobbs WR, Burke M, Christakis NA, Fowler JH: Online social integration is associated with reduced mortality risk. Proceedings of the National Academy of Sciences. 2016, 113(46): 12980-4. doi: $10.1073 /$ pnas. 1605554113

18. Kim DA, Benjamin EJ, Fowler JH, Christakis NA: Social connectedness is associated with fibrinogen level in a human social network. Proc. R. Soc. B 2016, 283: 20160958.

19. Greaves L, Hemsing N: Women and tobacco control policies: socialstructural and psychosocial contributions to vulnerability to tobacco use and exposure. Drug Alcohol Depend 2009, 104 Suppl 1: S12130 .

20. World Health Organization: Gender, women and the tobacco epidemic. 2010a. Geneva: World Health Organization.

21. Okoli C, Greaves L, Fagyas V: Sex differences in smoking initiation among children and adolescents. Public Health 2013, 127(1): 3-10.

22. Madeley J: Big business, poor peoples: the impact of transnational corporations on the world's poor. 1999 Palgrave Macmillan.

23. Graham H, Der G: Patterns and predictors of tobacco consumption among women. Health Educ Res 1999, 14(5): 611-8.

24. Thrift AP, Nancarrow H, Bauman AE: Maternal smoking during pregnancy among Aboriginal women in New South Wales is linked to social gradient. Australian and New Zealand Journal of Public Health 2011, 35(4): 337-342. doi: 10.1111/j.17536405.2011.00728.x

25. Action on Smoking and Health: "Tobacco and the Developing World" Fact sheet. August 2015. In: http://ash.org.uk/files/documents/ ASH_126.pdf (Accessed 14 January 2016).

26. Toll BA, Ling PM: The Virginia Slims identity crisis: an inside look at tobacco industry marketing to women. Tob Control 2005, 14(3): 172-80.

27. World Health Organization: Sifting the evidence: Gender and tobacco control. 2007. Geneva: World Health Organization, passim.
28. World Health Organization: Health for the world's adolescents: a second chance in the second decade: summary. 2014a. Geneva: World Health Organization.

29. Kokkevi A, Fotiou A, Kanavou E, Stavrou M, Richardson C: Smoking, alcohol, and drug use among adolescents in Greece 2015 update and secular trends $1984-2015$. Archives of Hellenic Medicine 2016, 33(2): 249-257.

30. Currie C, Molcho M, Boyce W, Holstein B, Torsheim T, Richter M: Researching health inequalities in adolescents: the development of the Health Behaviour in School-Aged Children (HBSC) family affluence scale. Social Science \& Medicine 2008, 66(6): 1429-1436.

31. Harakeh Z, de Looze ME, Schrijvers CT, van Dorsselaer SA, Vollebergh WA: Individual and environmental predictors of health risk behaviours among Dutch adolescents: the HBSC study. Public Health 2012, 126(7): 566-73. doi: 10.1016/j.puhe.2012.04.006

32. Askitopoulou V: "Supporting students to say no to smoking". In Round Table: "Smoking? Not for us!. A holistic school-based program to prevent tobacco use and addiction". Proceedings of the "6th International Congress on Brain and Behaviour". February 2014, Thessaloniki.

33. World Health Organization: Bollywood: victim or ally, a study on the portrayal of tobacco in Indian cinema. 2013b. Geneva: World Health Organization.

34. World Health Organization: Global progress report on implementation of the WHO Framework Convention on Tobacco Control. 2012. Geneva: World Health Organization.

35. Reddy KS, Yadav A, Arora M, Nazar GP: Integrating tobacco control into health and development agendas. Tob Control 2012, 21(2): 281-6.

36. Mentis AFA: Child migration: from social determinants of health to the development agenda and beyond. Medicine, Conflict and Survival 2016, 21:1-7.

37. Best CM, Sun K, de Pee S, Bloem MW, Stallkamp G, Semba RD: Parental tobacco use is associated with increased risk of child malnutrition in Bangladesh. Nutrition 2007, 23(10): 731-738.

38. Mentis AFA: To What Extent Are Greek Children Exposed to the Risk of a Lifelong, Intergenerationally Transmitted Poverty? Poverty \& Public Policy 2015, 7(4): 357-381.

39. Norman RE, Carpenter DO, Scott J, Brune MN, Sly PD: Environmental exposures: an underrecognized contribution to noncommunicable diseases. Reviews on Environmental Health 2013, 28(1): 59-65.

40. Sly PD, Neira M, Collman G, Carpenter DO, Landrigan PJ, Van Den Berg M, Barriga FD, Ruchirawat M, Laborde A, Pascale A, Heacock M, Dalmau MT, Suk WA: Networking to advance progress in children's environmental health. Lancet Glob Health 2014, 2(3): e129-30.

41. Gavidia T, Brune MN, McCarty KM, Pronczuk J, Etzel R, Neira M, Carpenter DO, Suk WA, Arnold RG, Ha EH, Sly PD: Children's environmental health-from knowledge to action. The Lancet 2011, 377: 1134-6.

42. Eriksen M, Mackay J, Ross H: The tobacco atlas (No. Ed. 4). 2013. American Cancer Society.

43. World Health Organization: 2010 global progress report on the implementation of the WHO Framework Convention on Tobacco Control. 2010b. Geneva: World Health Organization.

44. McKnight RH, Spiller HA: Green tobacco sickness in children and adolescents. Public Health Reports 2005, 120(6): 602-605.

45. Mund M, Louwen F, Klingelhoefer D, Gerber A: Smoking and pregnancy - a review on the first major environmental risk factor of the unborn. International Journal of Environmental Research and Public Health 2013, 10(12): 6485-6499. doi: 10.3390/ijerph10126485 


\section{Review Paper}

46. Campaign for Tobacco-free Kids: Press releases for 2001. In: http:// www.tobaccofreekids.org/press_releases/year/2001 (Accessed 11 January 2016).

47. Dixon A:The economics of tobacco and tobacco control, a development issue. In: http://ec.europa.eu/health/ph_determinants/life_style/ Tobacco/Documents/dixon.pdf (Accessed 15 January 2016).

48. Taylor A, Chaloupka FJ, Guindon E, Corbett M: The impact of trade liberalization on tobacco consumption. Tobacco control in developing countries 2000, 343-64.

49. Mamudu HM, Hammond R, Glantz S: Tobacco industry attempts to counter the World Bank report curbing the epidemic and obstruct the WHO framework convention on tobacco control. Social Science \& Medicine 2008, 67(11): 1690-1699. doi: 10.1016/j. socscimed.2008.09.062.

50. Bettcher D, Subramaniam C, Guindon E: Confronting the tobacco epidemic in an era of trade liberalization. 2001. Geneva: World Health Organization.

51. Fooks GJ, Gilmore AB: Corporate philanthropy, political influence, and health policy. PLoS One 2013, 8(11): e80864. doi: 10.1371/ journal.pone.0080864.

52. Amon JJ, Buchanan J, Cohen J, Kippenberg J: Child labor and environmental health: government obligations and human rights. Int J Pediatr 2012, 2012: 938306.

53. Human Rights Watch: World Report 2014. 2014. In: https://www. hrw.org/world-report/2014 (Accessed 10 January 2016).

54. United States Department of Labor: 2014 Findings on the Worst Forms of Child Labor. In: http://www.dol.gov/ilab/reports/childlabor/malawi.htm (Accessed 10 January 2016).

55. The Guardian: Child labour: the tobacco industry's smoking gun. 2011. In: http://www.theguardian.com/global-development/2011/ sep/14/malawi-child-labour-tobacco-industry (Accessed 11 January 2016).

56. Plan International: Malawi child tobacco pickers' '50-a-day' habit. 2009. In: www.plan-international.org (Accessed 11 January 2016).

57. Beaglehole R, Yach D: Globalisation and the prevention and control of non-communicable disease: the neglected chronic diseases of adults. The Lancet 2003, 362(9387): 903-908.

58. McCoy RS: So-called benefits of tobacco industry debunked. 1996. Utusan Konsumer, Mid-July.

59. Zhong F, Yano E: British American Tobacco's tactics during China's accession to the World Trade Organization. Tob Control 2007, 16(2): 133-7.

60. Hirschhorn N: Corporate social responsibility and the tobacco industry: hope or hype? Tobacco Control 2004, 13(4): 447-453.

61. Palazzo G, Richter U: CSR business as usual? The case of the tobacco industry. Journal of Business Ethics 2005, 61(4): 387-401.

62. Ulucanlar S, Fooks GJ, Gilmore AB: The policy dystopia model: An interpretive analysis of tobacco industry political activity. PLoS Med 2016, 13: e1002125. doi: 10.1371/journal.pmed.1002125

63. Zeltner T, Kessler DA, Martiny A, Randera F: Report of the Committee of Experts on Tobacco Industry Documents. 2000. Geneva: World Health Organization.

64. Warner KE: The economics of tobacco: myths and realities. Tobacco control 2000, 9(1): 78-89.

65. Chaloupka FJ, Yurekli A, Fong GT: Tobacco taxes as a tobacco control strategy. Tobacco Control 2012, 21 (2), 172-180.

66. World Health Organization: MPOWER: a policy package to reverse the tobacco epidemic. 2008b. Geneva: World Health Organization.

67. World Health Organization: 2014 Global progress report on implementation of the WHO Framework Convention on Tobacco Control. 2014b. Geneva: World Health Organization.

CONFLICT OF INTERESTS

All the authors have completed and submitted the ICMJE Form for Disclosure of Potential Conflicts of Interest and none were reported.

\section{FUNDING}

There was no source of funding for this research.

\section{PROVENANCE AND PEER} REVIEW

Not commissioned;

Externally peer reviewed 